\title{
The Seismic Intensity Monitoring System based on MEMS Seismic Intensity Instruments
}

\author{
Qiao Tan, Yunkai Guo and Zhitao Li \\ Key Laboratory of Crustal Dynamics, Institute of Crustal Dynamics, China Earthquake \\ Administration, Beijing, 100085, China \\ email, zhitao.lee@163.com
}

\section{Keywords: Earthquake, monitoring, Seismic Intensity Instrument}

Abstract. To mitigate the earthquake damage, casualties and economic losses, the capacities of disasters monitoring and ear warning need to be strengthened. For this reason, a seismic intensity monitoring system was put forward in this paper. The monitoring system is composed of seismic intensity instrument and seismic intensity rapid report center. The seismic intensity instrument which is the front end of the system can measure the ground motion acceleration and calculate the intensity value rapidly. The seismic intensity rapid report center is responsible for seismic intensity information acquisition, analysis, processing and dissemination automatically. Through the preliminary theory and experiment analysis, the system could meet the needs for emergency response.

\section{Introduction}

As China is one of the biggest developing countries, solely relying on enhancing the structures' anti-seismic capability to mitigate the disasters' losses is not wise and reality. When a damage earthquake occurs, how to obtain the influence information of a given area quickly and accurately is a very important scientific and technological problem to be solved at present.

Along with the improvement of the disaster monitoring capability, the seismic intensity rapid report and early warning technology has become a research focus in the field of international earthquake disaster reduction. The seismic intensity rapid report and early warning systems have been continuously developed in many countries and areas, which achieved significant effectiveness. The seismic intensity rapid report system obtains the seismic intensity of an earthquake by measuring the ground motion parameters rapidly. The seismic intensity monitoring system can obtain the information of hypocenter and distributed information about ground motion parameters. The information describes the distribution relationship of the seismic intensity objectively and accurately, which reduces the misjudgment rate impacted by uncertainty factors, improves the accuracy of the determination of seismic influence field. So the seismic intensity monitoring system can provide the objective evidence for rapid understanding earthquake situation, rapid decision-making and emergency response after the strong earthquake ${ }^{[1-3]}$.

In this paper, a seismic intensity monitoring system is presented. The monitoring system is composed of seismic intensity instruments and seismic intensity rapid report center ${ }^{[4]}$. The functions of seismic intensity instruments include recognizing the ground motion signal acquisition, analysising and processing, and calculating the intensity value. The seismic intensity rapid report center, which is based on Geographic Information System (GIS), can realize seismic intensity information acquisition, analysis, processing and dissemination automatically.

\section{Design of The Seismic Intensity Monitoring System}

Time matters greatly on the seismic intensity rapid report. In order to improve the emergency response capability and decrease its maintenance costs, some strategies are adopted such as, the seismic intensity monitoring system based on GIS, the main working procedure of this system is auto-triggered. On one hand, the seismic intensity instruments run automatic, on the other hand, the seismic intensity rapid report center receives the data from intensity instruments, and completes the 
acquisition, analysis, processing and dissemination automatically. The reporting business flow chart of the seismic intensity monitoring system is shown in figure1 as below.

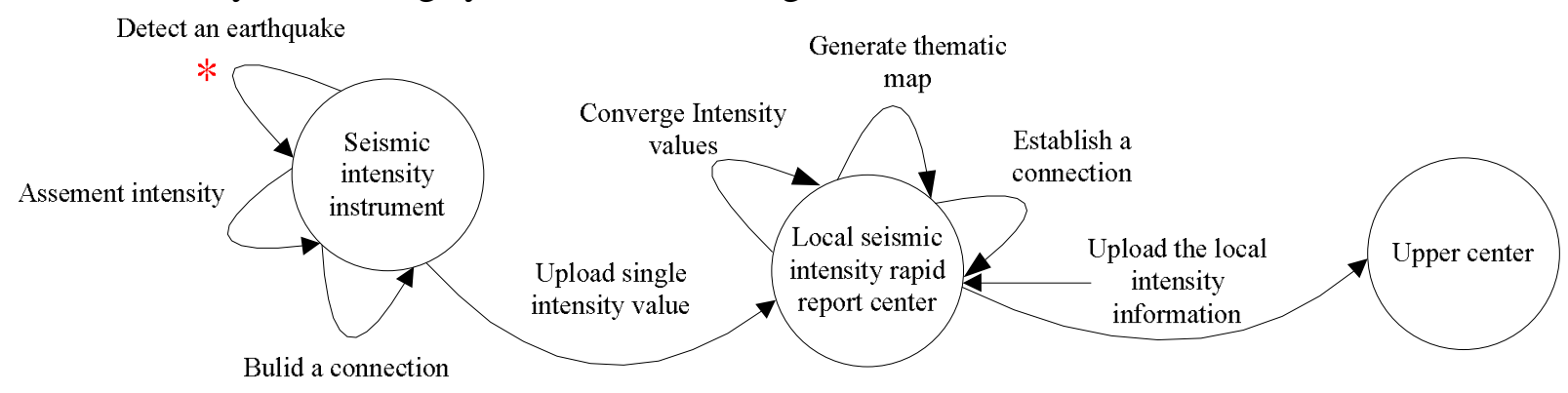

Figure 1: The reporting business flow chart of the seismic intensity monitoring system

As Shown in Figure 1, when an earthquake is detected, the seismic intensity instrument starts up and builds a connection to the local seismic intensity rapid report center automatically, then estimates the seismic intensity and uploads the single intensity value to the local center. Meanwhile the local center will converge to intensity values of the whole unattended area. After data from plurality of intensity instruments is received, the local center will renew its database and generate thematic maps based on the seismic intensity analysis algorithm. Then the intensity information will be uploaded to the upper center for further summarizing and processing.

\section{The Seismic Intensity Instrument}

As shown in Figure 2, the seismic intensity instrument is composed of the processor module, the data acquisition module (AD), the MEMS ${ }^{[5]}$ force-balanced accelerometer, IPv4/IPv6 network module, the $3 \mathrm{G}$ communication module, GPS module and the power management module.

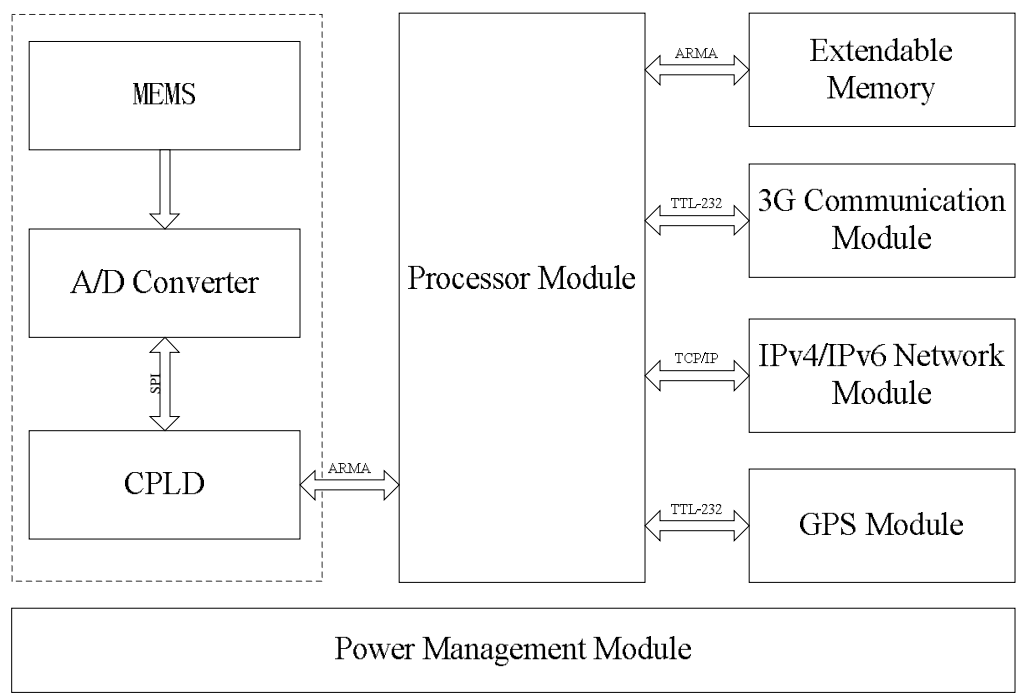

Figure 2: Overall structure of seismic intensity instrument

The processor module uses the low-power Cortex-M3 processor and integrates NOR and NAND flash. The force-balanced accelerometer, which is a variable capacitance accelerometer based on MEMS technology, can measure the ground motion in the X,Y,Z directions to provide data for the processor. In order to fulfill seismic signal acquisition, the data acquisition module uses the 24 bit $\Sigma$ - $\triangle$ modulation A/D converter which can dispel the signal distortion as well. GPS module is used for obtaining accurate longitude and latitude information. The IPv4 / IPv6 network module supports both IPv4 and IPv6 network protocol stack. The 3G communication module supports multi modes, such as WiFi, CDAM20001X EV-DO, TD-SCDMA, WCDMA 3G network etc. These two modules are used in conjunction to provide reliable data channel to upload seismic disaster information. 
The software system of seismic intensity instrument includes Linux operating system, hardware drivers and upper applications. The driver layer which is defined in the operating system provides software operation interface for each unit of the instrument. The main application program is composed of acceleration measurement, PGA/PGV/SI acquisition, seismic intensity estimation, seismic records reading / writing, earthquake alarm, fault alarm and so on.

\section{The Seismic intensity rapid report center}

\section{Block-based design}

The seismic intensity rapid report center has been developed top-down based on the structured and modular design method. Under the premise of comprehensive definition of the overall structure of the software system, application server, database server, GIS server and other functions are clearly defined. The functional block diagram of seismic intensity rapid report center is shown in figure 3 .

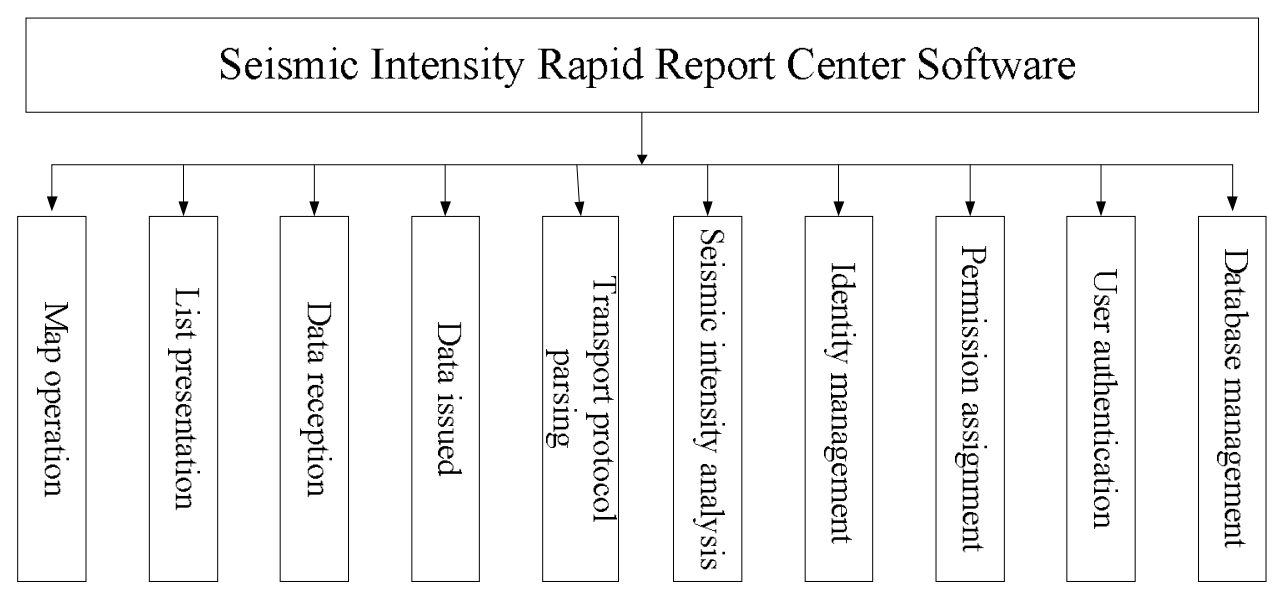

Figure 3: The functional block diagram of seismic intensity rapid report center

The entire software system according to the function can be divided into several blocks, for example map operation, list presentation, data reception, data issued, transport protocol parsing, seismic intensity analysis, identity management, permission assignment, user authentication, and the database management, etc.

The map operation module is composed mainly of GIS component which complete various map operations delivered by user and system. The main function of list presentation module is seismic intensity instrument displaying and other related operations. The data reception module is used for receiving the status and intensity information from the instrument. The data issued module is responsible for sending setting and function commands to the intensity instrument. The transport protocol parsing module implements the communication between the seismic intensity rapid report center and the seismic intensity instrument by protocol translation and conversion. When an earthquake occurs, the seismic intensity analysis module can generate the seismic thematic map based on the ground motion information uploaded by a plurality of intensity instruments. The identity management is the authentication unit which ensures the seismic intensity instrument to access the center software properly. User authentication module ensures that various users can login in the center software. Finally, the database management module is a functional unit for database backup, maintenance and management.

\section{The Business Procedure}

In addition to the daily management and human-computer interaction, the main business procedure of the seismic intensity rapid report center is seismic intensity information acquisition, analysis, processing and dissemination automatically. The main business procedure is shown in figure 4 . 


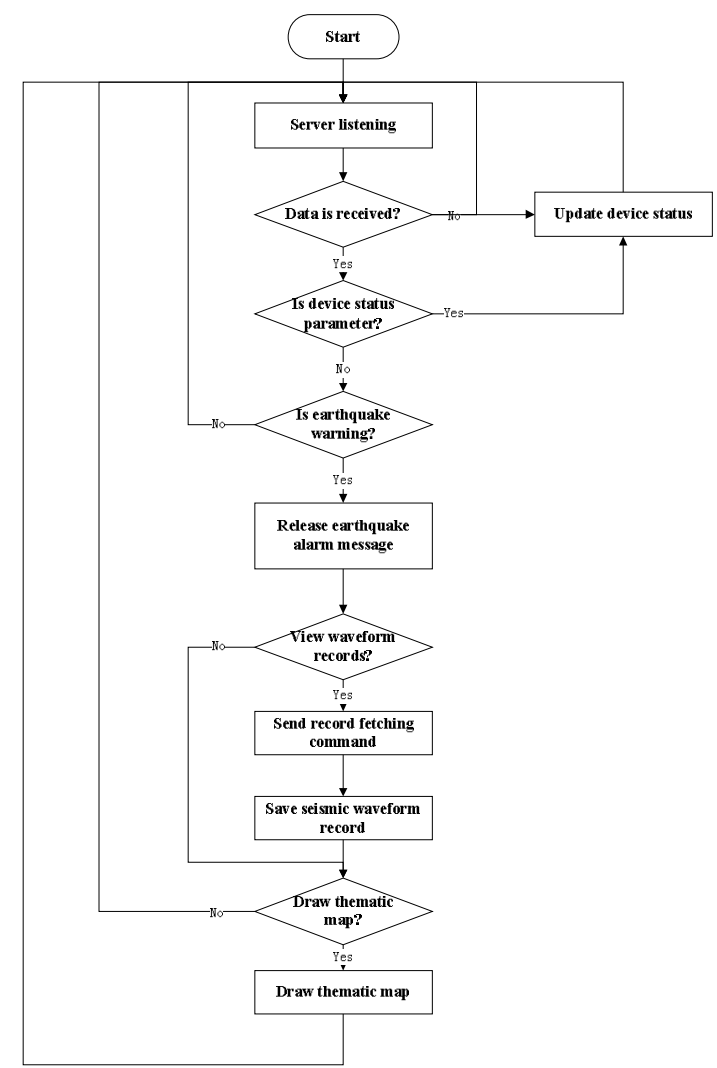

Figure 4: The business procedure of seismic intensity rapid report center

The server monitors the network port continuously to determine whether the data is received. After receiving the data, the process will judge the data type. If the data is device status parameter, the process will update the device status information and return. If the data is earthquake warning information, the process will release earthquake alarm message, and then draw thematic maps according seismic waveform data.

\section{The Seismic Intensity Analysis Algorithm}

The space model of the peak ground acceleration (PGA) and the spectral intensity (SI) is established by Kriging interpolation algorithm ${ }^{[6,7]}$ to generate thematic map of the seismic intensity distribution. The seismic intensity analysis process is shown in figure 5.

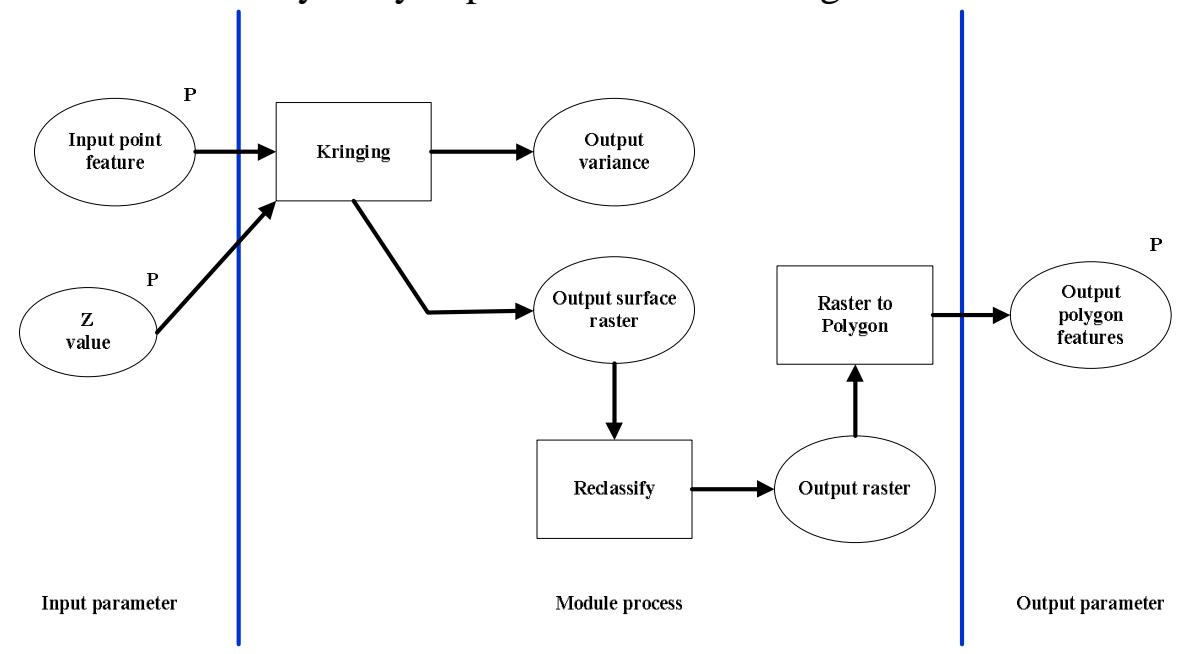

Figure 5: The analysis modeling flow diagram of seismic intensity algorithm

The input parameters and the model analysis tools of seismic intensity algorithm are separately shown in Table 1 and Table 2. 
Table 1: The seismic intensity algorithm

\begin{tabular}{lll}
\hline $\begin{array}{c}\text { No } \\
.\end{array}$ & \multicolumn{1}{c}{ Name } & \multicolumn{1}{c}{ Description } \\
\hline 1 & $\begin{array}{l}\text { Input point } \\
\text { features }\end{array}$ & $\begin{array}{l}\text { The input point set } \\
\text { The field name of the peak ground acceleration(PGA) and the spectral } \\
\text { intensity(SI) }\end{array}$ \\
2 & Z value field & $\begin{array}{l}\text { Kriging interpolation algorithm, Interpolating the peak ground } \\
\text { acceleration(PGA) according to the field name and generate a raster } \\
\text { Reclassifying each peak ground acceleration(PGA) and spectral } \\
\text { intensity(SI) within the raster } \\
\text { Converting raster to polygon }\end{array}$ \\
\hline
\end{tabular}

According to the recent research about the Chinese Seismic Intensity Scale which includes the peak ground acceleration (PGA) and the spectral intensity(SI), the seismic intensity is reclassified. The Chinese Seismic Intensity Scale is showed below:

Table 2: The Chinese Seismic Intensity Scale

\begin{tabular}{ccc}
\hline Seismic Intensity & PGA $\left(\mathrm{cm} / \mathrm{s}^{2}\right)$ & SI $(\mathrm{cm})$ \\
\hline V & $22-44$ & $3.2-6.7$ \\
\hline VI & $45-89$ & $6.8-14.0$ \\
\hline VII & $90-177$ & $14.1-29.1$ \\
\hline VIII & $178-353$ & $29.2-107.8$ \\
\hline IX & $354-707$ & $107.9-$ \\
\hline$X$ & $708-1414$ & - \\
\hline
\end{tabular}

\section{Experiments and Results}

To show the performances of the seismic intensity monitoring system, a series of testing experiments and simulation were carried out. And the results showed that the system could meet the needs for mitigating the disasters, casualties and economic losses.

\section{Test of the seismic intensity instrument}

The outputs of the seismic intensity instrument are recorded at different frequency points $(2,5,10$ and $20 \mathrm{~Hz}$ ). The test results are shown as below:

Table 3: The frequency response characteristic of seismic intensity instrument

\begin{tabular}{lllll}
\hline Frequency $(\mathrm{Hz})$ & 2 & 5 & 10 (Reference) & 20 \\
Sensitivity $(\mathrm{V} / \mathrm{g})$ & 2.597 & 2.578 & 2.578 & 2.580 \\
Deviation $(\%)$ & 0.04 & 0.00 & 0.00 & 0.08 \\
\hline
\end{tabular}

The test results indicate that the seismic intensity instrument could measure ground motion with sufficient accuracy and sensitivity deviations were less than $0.1 \%$.

\section{Simulation of the seismic intensity monitoring system}

In the whole system, a single seismic intensity instrument could upload the intensity data within 5 minutes; the seismic intensity rapid report center could aggregate the intensity data of the unattended region within 10 minutes, and calculate the rapid report message, generate the thematic maps within 20 minutes. The thematic map was shown below: 


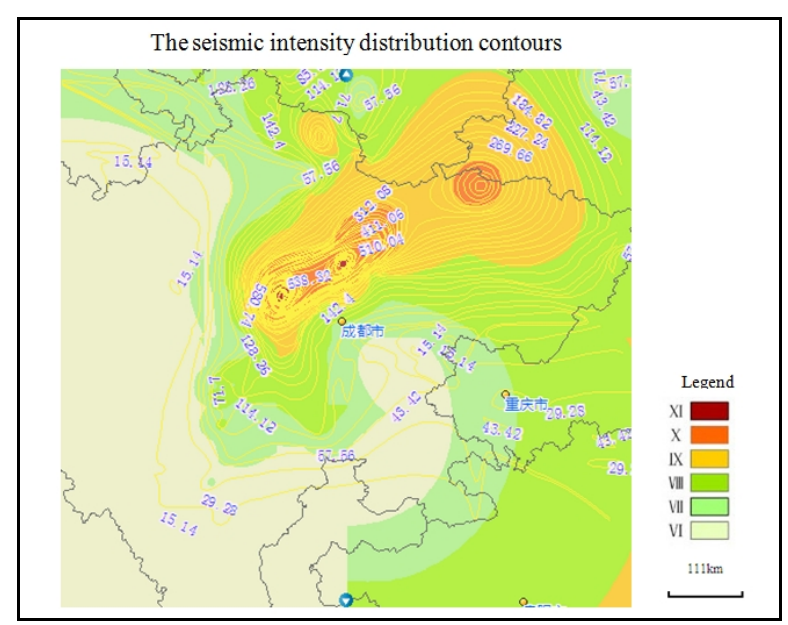

Figure 6: The seismic intensity distribution contours

The data sets of Wenchuan main shock were used in this study. The thematic map as shown in figure 7 was a seismic intensity distribution contours. According to this map, the earthquake relief could be distributed more reasonably.

\section{Conclusion}

The seismic intensity monitoring system which is composed of seismic intensity instruments and seismic intensity rapid report center, can implement intensity data acquisition accurately and effectively through multiple communication network. Meanwhile it can provide further features of data analysis and issuing which contributes to the rapid response to sudden earthquake events.

\section{Acknowledgement}

This work was financially supported by the research grant from Institute of Crustal Dynamics, China Earthquake Administration (ZDJ2012-18).

\section{References}

[1] Fu Jihua, Li Zhitao, Tan Qiao, Wang Jianjun. An earthquake emergency alarm system for power plants based on programmable automation controller. Applied Mechanics and Materials, 2014, 986-987: 1178-1182

[2] Yih-Min Wu, Nai-Chi Hsiao, Ta-Liang Teng, Tzay-Chyn Shin. Near Real-Time Seismic Damage Assessment of the Rapid Reporting Systems[J]. TAO ,2002, 13(3):313-324.

[3] Kanamori H., Hauksson E., Heaton T. Real-time seismology and earthquake hazard mitigation. Nature, 1997, 390(4): 461-464

[4] Guo Yunkai, Tan Qiao, Li Zhitao, Fu Jihua, Xiao Ke.Design of Seismic Intensity Rapid Report Platform. Advance in Computer Science Research, 2014(1): 381-386.

[5] J. R. Evans, R. M. Allen, etc. Performance of Several Low-Cost Accelerometers [J]. Seismological Research Letters, January/February 2014,147-158.

[6] Davis,John C.Statistics and Data Analysis in Geology.3rd Edition. NewYork:John Wiley\&Sons,Inc, 2002.57-61.

[7] X Seiichi, T Hirokazu, T Yuuichi, et al. Spatial Interpolation of Weather SensorData on P2P Network. In: Proc of International Conference on Complex Intelligent and Software Intensive Systems. New York, 2009, 1-69 